INRA Prod. Anim., 2013, 26 (1), 3-12

\title{
Utilisation des huiles essentielles en alimentation des volailles. 1. Performances de croissance et règlementation
}

\author{
F. ALLEMAN', I. GABRIEL ${ }^{2}$, V. DUFOURCQ $Q^{1}$,F. PERRIN ${ }^{1}$, J.-F. GABARROU \\ ${ }^{1}$ Ecole d'Ingénieurs de Purpan, 75 voie du TOEC, F-31076 Toulouse, France \\ 2 INRA, UR83 Recherches Avicoles, F-37380 Nouzilly, France
}

Courriel : fabien.alleman@purpan.fr

\begin{abstract}
Depuis l'interdiction des «Antibiotiques Facteurs de Croissance » en 2006, les huiles essentielles, comme d'autres extraits végétaux à connotation « naturelle » connaissent un développement important. L'alimentation animale, tout particulièrement en aviculture, n'échappe pas à cette tendance. Mais les extraits végétaux comme les huiles essentielles sont difficiles à caractériser, leur composition chimique est complexe, leur efficacité variable. Avec une dizaine d'années de recul, le moment est venu de faire le point sur l'utilisation régulière de ces huiles essentielles en aviculture.
\end{abstract}

L'apparition récurrente de problèmes de santé publique liés aux bactéries résistantes aux antibiotiques a conduit les autorités européennes à interdire au $1^{\text {er }}$ janvier 2006 les antibiotiques comme facteurs de croissance en alimentation animale (article 11-2 du règlement (CE) $\left.\mathrm{n}^{\circ} 2003 / 1831\right)$. Les industriels de l'alimentation animale se sont alors efforcés de rechercher des alternatives à ces molécules, désormais bannies de la liste des additifs pour les animaux d'élevage.

Les produits à base de plantes, déjà utilisés pour leurs effets sur les performances zootechniques chez les volailles depuis les années 1990 (Brenes et Roura 2010), ont vu leur utilisation se développer fortement. Ainsi, plusieurs nouveaux mélanges d'Huiles Essentielles (HE) ou de composés synthétiques (associés ou non à d'autres produits tels que les épices), ont fait leur apparition sur le marché mondial.

L'objectif de cet article est de faire le point sur l'utilisation des HE en alimentation des volailles. Après un bref rappel de la définition d'une HE, de leur composition chimique et de leur statut réglementaire en Europe, nous présenterons les produits les plus décrits dans la littérature pour leurs effets sur les performances zootechniques des volailles, et la toxicité éventuelle de leurs principes actifs.

\section{1 / Qu'est-ce qu'une huile essentielle?}

\section{1 / Définition}

Selon la Pharmacopée Européenne (2011), une HE est un "produit odorant, généralement de composition complexe, obtenu à partir d'une matière première végétale botaniquement définie, soit par entraînement par la vapeur d'eau, soit par distillation sèche, ou par un procédé mécanique approprié sans chauffage. L'huile essentielle est le plus souvent séparée de la phase aqueuse par un procédé physique n'entraînant pas de changement significatif de sa composition ». En pratique, il est possible d'obtenir une HE à partir de la plante entière ou bien seulement à partir de certaines parties de la plante telle les fleurs, bourgeons, grains, feuilles, bois, écorce, fruits, racines, tiges et brindilles (Brenes et Roura 2010).

On peut aujourd'hui ainsi obtenir plus de 3000 sortes d'HE, dont 300 sont commercialisées à des fins très diverses (en pharmacie, parfumerie, cosmétique), comme produits phytosanitaires, comme sources d'arômes (pour masquer l'odeur des produits ménagers ou comme arôme alimentaire) et enfin en alimentation humaine et animale (Brenes et Roura 2010). Les propriétés antibactériennes de certaines d'entre elles peuvent également justifier leur utilisation.

Dans le domaine des productions animales, les HE sont principalement utilisées pour améliorer les performances zootechniques (vitesse de croissance, Indice de Consommation (IC), niveau de l'ingéré, digestibilité des aliments, statut sanitaire des animaux). De manière plus générale, les propriétés des $\mathrm{HE}$ identifiées jusqu'à présent sont extrêmement variées (Brenes et Roura 2010), au premier rang desquelles on citera les propriétés antibactériennes (Demir et al 2005) et antioxydantes (Botsoglou et al 2003), les effets de stimulation du tractus digestif (Jang et al 2007), les propriétés antivirales (Giannenas et al 2003), antimycosiques (Soto Mendivil et al 2006), antiparasitaires (Pandey et al 2000), hypolipémiantes (Konjufca et al 1997), inhibitrices d'odeurs (Smith et al 2009) et insecticides (Konstantopoulou et al 1992). Ces propriétés seront largement évoquées dans la troisième partie de cet article.

\section{2 / Composition chimique des huiles essentielles : complexité et variabilité}

Une HE est un mélange complexe d'un grand nombre de composés liposolubles différents (Dorman et Deans 2000). Différents types de classification existent. On peut considérer qu'elles sont 
Tableau 1. Composition chimique de l'huile essentielle de thym (\%).

Pour chaque molécule, la valeur minimale figure en noir gras et la valeur maximale en rouge gras.

\begin{tabular}{|l|c|c|c|c|}
\hline Molécule & $\begin{array}{c}\text { Soto Mendivil } \\
\text { et al (2006) }\end{array}$ & $\begin{array}{c}\text { Porte } \\
\text { et Godoy (2007) }\end{array}$ & $\begin{array}{c}\text { Imelouane } \\
\text { et al (2009) }\end{array}$ & $\begin{array}{c}\text { Martino } \\
\text { et al (2009) }\end{array}$ \\
\hline Thymol & 16,6 & 44,7 & $\mathbf{0 , 2 4}$ & 48,9 \\
\hline Camphre & 3 & $\mathbf{0}$ & 38,54 & 0,2 \\
\hline Bornéol & $\mathbf{2 8 , 4}$ & $\mathbf{0 , 5}$ & 4,92 & 1,7 \\
\hline p-cymène & 2,4 & 18,6 & $\mathbf{1 , 1 9}$ & 19 \\
\hline Camphène & 6,9 & $\mathbf{0 , 3}$ & 17,19 & 0,8 \\
\hline Y- terpinène & 1,7 & 16,5 & $\mathbf{0 , 5 5}$ & 4,1 \\
\hline $\begin{array}{l}\text { Carvacrol éther } \\
\text { de méthyle }\end{array}$ & $\mathbf{9 , 6}$ & $\mathbf{0}$ & $\mathbf{0}$ & 1,7 \\
\hline $\boldsymbol{\alpha}$-pinène & 4,2 & $\mathbf{0 , 8}$ & $\mathbf{9 , 3 5}$ & 1,2 \\
\hline $\boldsymbol{\alpha}$-humulène & 6,4 & $\mathbf{0}$ & $\mathbf{0}$ & 0,3 \\
\hline 1,8-cinéole & 0,1 & $\mathbf{0}$ & $\mathbf{5 , 4 5}$ & 0,7 \\
\hline Carvacrol & $\mathbf{5}$ & 2,4 & $\mathbf{0}$ & 3,5 \\
\hline
\end{tabular}

composées de terpénoïdes, eux-mêmes classés en fonction de leur nombre d'unités isoprène (Loomis et Croteau 1980). Ainsi, on peut différentier les monoterpènes qui représentent la très grande majorité de ces molécules, les diterpènes, les triterpènes, les tétraterpènes, les sesterpènes, les sesquiterpènes, et les polyterpènes. La structure de ces molécules peut être de type linéaire ou cyclique, et comporter des fonctions chimiques très différentes (éthanolique, aldéhydique, cétonique...). Par ailleurs, parmi ces nombreux composants, tous ne sont pas encore identifiés.

Une caractéristique remarquable des HE est la grande variabilité de leur teneur en principes actifs. Les conditions agronomiques comme la nature du sol, l'origine géographique, le climat, l'al- titude influencent la composition des $\mathrm{HE}$ de la plante. Ainsi, pour une espèce donnée, plusieurs " chémotypes » (i.e. des variations de teneurs en métabolites secondaires) peuvent être distingués chimiquement. Les compositions varient également selon l'état physiologique de la plante, tel que son âge et sa maturité (stade et période de récolte), ainsi que l'organe de la plante utilisé (feuille, fleur, racine...) pour extraire l'HE.

A cette variabilité inhérente à la plante, s'ajoute une variabilité liée aux traitements de la plante après sa récolte (séchage, méthode d'extraction). La nature des solvants et les conditions d'extraction (concentration et composition des solvants d'extraction, temps de contact, température...) sélectionnent des composés variables et peuvent conduire à des produits de niveau d'activité et de propriétés différents.

La composition de ces HE peut ensuite varier selon les conditions de conservation du fait de la volatilité relative de certains composants dont les concentrations peuvent diminuer au cours du temps. Lors de la fabrication des aliments, des interactions avec des constituants des pré-mélanges ou de l'aliment, ou l'application de procédés technologiques (chauffage, agglomération...) peuvent conduire à la réduction (voire la disparition) de certains composants ou à leur modification structurale. Pour protéger ces molécules, des procédés d'encapsulation peuvent être effectués.

Pour chaque HE, les teneurs de plusieurs molécules sont liées entre elles. En effet, quand un composé (comme le thymol pour l'huile de thym) est présent en quantité importante, la concentration d'un autre composé chimique (comme le para-cymène) s'en trouve réduite et vice et versa. Ceci pourrait s'expliquer par le fait que ces composés possèdent une voie métabolique semblable et, qu'en fonction de différents facteurs liés à la variété, au pédoclimat et au stade de récolte de la plante considérée, certains composés se substituent à d'autres (voie métabolique).

A titre d'illustration (tableaux 1, 2 et 3), si les principes actifs principaux de l'HE de thym semblent être liés à la présence du thymol, du camphre et du bornéol, la concentration en thymol dans l'HE de thym varie de 0,24 (Imelouane et al 2009) à 48,9\% (Porte et Godoy 2007). De même, parmi les principes actifs principaux de l'HE de romarin

Tableau 2. Composition chimique de l'huile essentielle d'origan (\%).

Pour chaque molécule, la valeur minimale figure en noir gras et la valeur maximale en rouge gras.

\begin{tabular}{|c|c|c|c|c|c|c|c|c|c|}
\hline \multirow[t]{2}{*}{ Molécules } & \multicolumn{3}{|c|}{$\begin{array}{l}\text { Filippo d'Antuono } \\
\text { et al (2000) }\end{array}$} & \multicolumn{3}{|c|}{$\begin{array}{c}\text { Azizi } \\
\text { et al (2008) }\end{array}$} & \multicolumn{3}{|c|}{$\begin{array}{l}\text { Sokovic } \\
\text { et al (2009) }\end{array}$} \\
\hline & origan 1 & origan 2 & origan 3 & origan 1 & origan 2 & origan 3 & origan 1 & origan 2 & origan 3 \\
\hline Carvacrol & 18,47 & 1,23 & 0,14 & 77,4 & 74,9 & 70 & 21,89 & 6,45 & 0,46 \\
\hline Linalool & 0,58 & 28,18 & 3,92 & 0,1 & 0 & 0 & 2,87 & 4,1 & 12,5 \\
\hline Thymol & 21,65 & 10,88 & 0,72 & 0,3 & 3,7 & 0,3 & 18,21 & 26,75 & 3,24 \\
\hline $\mathrm{Y}$-terpinène & 18,2 & 4,85 & 1,82 & 8,1 & 8,2 & 9,5 & 2,38 & 4,59 & 4,9 \\
\hline Linalyl acetate & 0 & 0 & 0 & 0 & 0 & 0 & 0,48 & 1,2 & 15,9 \\
\hline Caryophyllène oxyde & 0,6 & 3,56 & 15,82 & 0,1 & 0 & 0,1 & 1,01 & 0,6 & 1,2 \\
\hline a-terpinéol & 0,12 & 0,88 & 2,71 & 0,2 & 0,1 & 0 & 0,22 & 15,1 & 3,9 \\
\hline E-caryophyllène & 6,02 & 13,24 & 6,68 & 3 & 3,1 & 2,8 & 3,72 & 2,11 & 4,29 \\
\hline Germacrène-d-4-ol & 0,33 & 1,49 & 10,69 & 0,1 & 0,6 & 0,8 & 0 & 0 & 0 \\
\hline V-muurolène & 3,99 & 10,48 & 8,72 & - & - & - & 4,48 & 2,59 & 3,61 \\
\hline$\rho$-cymène & 9,37 & 2,18 & 2,67 & - & - & - & 2,81 & 1,25 & 2,01 \\
\hline$\beta$-bourbonène & 0,15 & 0,46 & 8,81 & - & - & - & 0,33 & 0,2 & 0,6 \\
\hline
\end{tabular}


Tableau 3. Composition chimique de l'huile essentielle de romarin (\%).

Pour chaque molécule, la valeur minimale figure en noir gras et la valeur maximale en rouge gras.

\begin{tabular}{|c|c|c|c|c|c|c|c|c|c|}
\hline \multirow[t]{2}{*}{ Molécule } & \multicolumn{7}{|c|}{$\begin{array}{c}\text { Santoyo } \\
\text { et al (2005) }\end{array}$} & \multirow[t]{2}{*}{$\begin{array}{c}\text { Jamshidi } \\
\text { et Afzali (2009) }\end{array}$} & \multirow[t]{2}{*}{$\begin{array}{c}\text { Bousbia } \\
\text { et al (2009) }\end{array}$} \\
\hline & $\begin{array}{c}\text { Romarin } \\
1 \\
\end{array}$ & $\begin{array}{c}\text { Romarin } \\
2 \\
\end{array}$ & $\begin{array}{c}\text { Romarin } \\
3 \\
\end{array}$ & \begin{tabular}{|c|} 
Romarin \\
4 \\
\end{tabular} & $\begin{array}{c}\text { Romarin } \\
5 \\
\end{array}$ & $\begin{array}{c}\text { Romarin } \\
6 \\
\end{array}$ & $\begin{array}{c}\text { Romarin } \\
7 \\
\end{array}$ & & \\
\hline Y-pinène & 8,11 & 7,07 & 13,44 & 8,28 & 20,95 & 19,67 & 70,6 & 43,9 & 10 \\
\hline Camphre & 18,64 & 17,88 & 21,88 & 24,57 & 20,95 & 21,05 & 0 & 2,4 & 12 \\
\hline 1,8-cinéole & 18,03 & 21,28 & 21,98 & 19,07 & 22,23 & 21,68 & 0 & 11,1 & 50 \\
\hline Verbénone & 13,63 & 11,98 & 14,31 & 16 & 12,52 & 13,4 & 1,5 & 2,6 & 0 \\
\hline Camphène & 2,24 & 2,88 & 0 & 0 & 0 & 0 & 2,6 & 8,6 & 3,7 \\
\hline$\alpha$-terpinène & 4,84 & 7,83 & 0,49 & 0 & 0,35 & 0,75 & 0 & 0,4 & 0,4 \\
\hline Bornéol & 6,18 & 5,66 & 6,79 & 7,7 & 6,08 & 6,45 & 0 & 3,4 & 3,4 \\
\hline $\begin{array}{l}\text { trans- } \\
\text { Caryophylléne }\end{array}$ & 6,03 & 4,9 & 5,3 & 6,24 & 4,67 & 4,8 & 0,92 & 1,6 & 3,9 \\
\hline Bicycloheptane & 0 & 0 & 0 & 0 & 0 & 0 & 0 & 0 & 6,5 \\
\hline Abietatriène & 0,36 & 0,3 & 0,21 & 0,37 & 0 & 0 & 5,16 & 0 & 0 \\
\hline Y-terpinéol & 4,5 & 3,72 & 4,38 & 4,91 & 3,83 & 4,04 & 0 & 0,4 & 0,9 \\
\hline
\end{tabular}

comme l' $\alpha$-pinène, le camphre et le 1,8 cinéol, le 1,8-cinéol peut varier en concentration dans l'HE de 0 (Santoyo et al 2005) à 50\% (Mathlouthi et al 2009). Enfin, pour l'HE d'origan, la concentration en carvacrol dans l'HE varie de 0,14 (Filippo d'Antuono et al 2000) à 77,4\% (Azizi et al 2008).

Bien que tout à fait « naturelle », cette variabilité de la composition des HE est assez peu compatible avec l'obligation de garantir la composition des additifs au sens du règlement $(\mathrm{CE}) \mathrm{n}^{\circ} 2003 / 1831$. $\mathrm{C}^{\prime}$ 'est pourquoi les fabricants préfèrent utiliser des produits de synthèse dits « identiques naturels » pour assurer une composition plus standardisée et reproductible.

\section{3 / Statut réglementaire des huiles essentielles en alimentation animale}

Avant la mise en application du règlement (CE) $n^{\circ}$ 2003/1831 (Commission européenne 2003), les HE en alimentation animale appartenaient à la catégorie des « Substances aromatiques et apéritives » dans le règlement $(\mathrm{CE}) \mathrm{n}^{\circ} 70 /$ 524. Par ce simple classement, elles étaient « naturellement » autorisées à la vente (AFSSA 2007).

Depuis 2003, les produits à base d'HE sont régis par le règlement $(\mathrm{CE}) \mathrm{n}^{\circ} 2003 /$ 1831. Ce règlement a, entre autres, imposé que tous les additifs déjà autorisés fassent l'objet d'un nouveau dépôt de dossier avant le 7 novembre 2010 afin d'être réévalués par l'AESA (Agence Européenne de Sécurité Alimentaire). Ainsi, chaque fabricant de produit à base d'HE a donc été conduit à positionner son (ou ses) produit(s) dans l'une des cinq catégories d'additifs définies dans le règlement ( $\mathrm{cf}$. encadré) et déposer le(s) dossier(s) d'autorisation correspondant(s).

Le contenu du dossier d'autorisation (i.e. les éléments scientifiques à fournir) pour chacune des cinq catégories d'additifs est donné dans le règlement (CE) $n^{\circ} 2008 / 429$ (Commission Européenne 2008). Le choix de positionner un additif dans une catégorie ou une autre peut ainsi faire varier sensiblement le nombre d'études à fournir, en particulier dans la partie «Efficacité », mais dans le même temps, permettre d'allonger la liste des allégations autorisées sur l'étiquette.

A l'heure actuelle, le nombre exact de dossiers déposés à l'AESA n'est pas connu, mais il s'élèverait à « plusieurs centaines $»$.

\section{2 / Les principales huiles essentielles utilisées en ali- mentation des volailles}

En aviculture, la plupart des études portant sur les HE concernent le poulet de chair et, dans une moindre mesure, la poule pondeuse. Cependant, il existe également quelques références sur la caille et la dinde.

Pour un grand nombre de publications relatant les effets de mélanges d'HE, la nature, la composition et les doses ne sont pas renseignées. Ainsi, il est difficile, voire impossible, d'établir le lien entre les performances observées et la teneur de chaque composant du mélange, les phénomènes de synergie ou d'antagonisme entre leurs composés étant aujourd'hui encore peu décrits.

Les HE de thym, d'origan et de romarin sont les principales HE pour lesquelles des effets zootechniques sont les mieux

Encadré. Article 6 du règlement CE 2003/1831: les cinq catégories d'additifs pour l'alimentation animale.

Selon leurs fonctions et leurs propriétés, les additifs pour l'alimentation animale sont classés dans une ou plusieurs des catégories suivantes:

a) Additifs technologiques : toutes les substances ajoutées aux aliments pour animaux à des fins technologiques;

b) Additifs sensoriels : toutes les substances qui, ajoutées à l'alimentation animale, améliorent ou modifient les propriétés organoleptiques des aliments pour animaux ou les caractéristiques visuelles des denrées alimentaires issues d'animaux;

c) Additifs nutritionnels ;

d) Additifs zootechniques : tous les additifs utilisés pour influencer favorablement les performances des animaux en bonne santé ou l'environnement ;

e) Coccidiostatiques et histomonostatiques. 
et les plus fréquemment rapportés. Ceci pourrait s'expliquer par le fait que leur commercialisation est assujettie au dépôt d'un dossier d'autorisation que l'industriel doit financièrement amortir (cf. § 1.3). Ainsi, placer sur le marché un produit en deçà de son prix d'intérêt, mais permettant au fabricant de s'assurer un retour sur investissement dans un délai raisonnable, impose de disposer d'une matière première à bas prix et en quantité disponible suffisante. Or, le thym, l'origan et le romarin sont trois des quatre plantes majeures entrant dans la fabrication des mélanges commercialisés sous le nom d' «Herbes de Provence». Cette production importante destinée principalement à la consommation humaine permet aux fabricants d'HE d'accéder à une matière première abondante. De plus, ces plantes sont très riches en HE et présentent des rendements d'extraction élevés (de l'ordre de $1 \%$, contre moins de $0,5 \%$ pour d'autres plantes aromatiques). Ainsi, un additif ajouté à moins de 200 ppm dans l'aliment et contenant $10 \%$ d'HE permettrait, d'après les calculs, de proposer une matière active à un coût inférieur à 5 euros par tonne d'aliment traité, ce qui est la limite acceptable en industrie des aliments du bétail pour ce genre de produits.

\section{3 / Efficacité des huiles essen- tielles en alimentation des volailles}

Comme pour l'effet d'autres alternatives aux antibiotiques, les HE présentent une grande variabilité d'effets biologiques rapportés. Ceci s'explique par la grande disparité des conditions dans lesquelles sont étudiés ces mélanges: souches et stades physiologiques des animaux, conditions d'élevage plus ou moins favorables, doses (Elhusseiny et al 1980) et périodes d'utilisation (démarrage, croissance-finition, ou tout au long de l'élevage) (Elhusseiny et al 1980, Tekeli et al 2006). A cela s'ajoute la complexité et la variabilité de leur composition comme cela a été indiqué précédemment. Ainsi, les trois types d'HE les plus utilisés en alimentation animale (thym, origan et romarin) présentent une grande variabilité d'effets zootechniques.

Afin d'améliorer la précision de notre revue, nous avons compilé les résultats de plusieurs publications dans une base de données unique, et réalisé une métaanalyse simple. Pour cela, seuls les articles présentant un traitement témoin sans HE ont été retenus. De même, les traitements incluant plusieurs extraits végétaux différents ont été exclus. Les données compilées ont, dans un second temps, été analysées via un test de "Student» appariant la moyenne de chaque traitement à son traitement témoin. Cette méthode a été répétée pour chacune des HE étudiées.

\section{1 / L'huile essentielle de thym}

Les publications traitant des effets de l'HE de thym sur les performances de croissance du poulet montrent des effets contrastés. Bolukbasi et al (2006) et Cross et al (2007) ont observé une augmentation de la consommation alimentaire et une dégradation de l'IC alors que $\mathrm{Al}$ Kassie (2009) et Tekeli et al (2006) ont noté une amélioration des niveaux des indices de croissance et de consommation malgré une baisse du niveau de consommation. En revanche, Lee et al (2003) et Najafi et Torki (2010) n'ont observé aucun effet significatif.

La méta-analyse concernant l'emploi de l'HE de thym (tableau 4) chez le poulet de chair ne met pas en évidence d'effet significatif de ce produit sur les performances de croissance. Une simple tendance $(P=0,06)$ de l'effet des HE de thym sur le Gain Moyen Quotidien (GMQ) a pu être observée.

Avec de l'HE de thym à $0,7 \%$ dans l'aliment, des augmentations significatives du poids de carcasse et du rendement en carcasse ont été rapportées chez le poulet, mais l'augmentation n'est plus significative à des doses plus faibles de 0,1 et 0,3\% (Fotea et al 2009). Cependant, d'autres travaux montrent l'absence d'effet sur le rendement en carcasse de l'emploi des feuilles de thym broyées $(10 \mathrm{~g} / \mathrm{kg})$ chez le canard (AbouSekken et al 2007) ou avec 5 et $10 \mathrm{~g} / \mathrm{kg}$ chez le poulet (Toghyani et al 2010), ce qui représente l'équivalent de $0,01 \%$ d'HE. Cette faible dose pourrait expliquer l'absence d'effet observé.

Chez le poulet de chair, Fotea et al (2009) n'observent pas de modification de la composition chimique (humidité,

Tableau 4. Effets des huiles essentielles de thym, d'origan et de romarin sur les performances de croissance du poulet de chair.

\begin{tabular}{|c|c|c|c|c|c|}
\hline & Témoin & HE & $P$ & $\begin{array}{c}\% \text { de } \\
\text { variation }\end{array}$ & Références \\
\hline \multicolumn{6}{|l|}{ Thym } \\
\hline \begin{tabular}{|l|} 
Indice de \\
consommation \\
\end{tabular} & $1,784 \pm 0,085$ & $1,744 \pm 0,084$ & 0,102 & $-2,29$ & \multirow{3}{*}{$\begin{array}{l}\text { Lee et al (2003), Tekeli et al (2006), } \\
\text { Bolukbasi et al (2006), Cross et al (2007), } \\
\text { Al Kassie (2009), Najafi et Torki (2010) }\end{array}$} \\
\hline $\begin{array}{l}\text { Consommation } \\
\text { alimentaire }(\mathrm{g} / \mathrm{j})\end{array}$ & $94,0 \pm 13,0$ & $96,0 \pm 13,6$ & 0,124 & $+2,14$ & \\
\hline $\begin{array}{l}\text { Gain moyen } \\
\text { quotidien }(\mathrm{g} / \mathrm{j})\end{array}$ & $50,7 \pm 5,4$ & $52,5 \pm 5,8$ & 0,060 & $+3,53$ & \\
\hline \multicolumn{6}{|l|}{ Origan } \\
\hline \begin{tabular}{|l|} 
Indice de \\
consommation \\
\end{tabular} & $1,607 \pm 0,053$ & $1,578 \pm 0,050$ & 0,011 & $-1,84$ & \multirow{3}{*}{$\begin{array}{l}\text { Tekeli et al (2006), Basmacioglu et al } \\
(2004), \text { Halle et al (2005), Cross et al } \\
(2007), \text { Symeon et al (2009), Basmacioglu } \\
\text { et al (2010) }\end{array}$} \\
\hline $\begin{array}{l}\text { Consommation } \\
\text { alimentaire }(\mathrm{g} / \mathrm{j})\end{array}$ & $88,0 \pm 10,2$ & $86,6 \pm 10,0$ & 0,072 & $+1,54$ & \\
\hline \begin{tabular}{|l|} 
Gain moyen \\
quotidien $(\mathrm{g} / \mathrm{j})$
\end{tabular} & $43,8 \pm 3,4$ & $44,9 \pm 3,6$ & 0,006 & $+2,38$ & \\
\hline \multicolumn{6}{|l|}{ Romarin } \\
\hline \begin{tabular}{|l|} 
Indice de \\
consommation \\
\end{tabular} & $1,942 \pm 0,113$ & $1,867 \pm 0,080$ & 0,066 & $-4,02$ & \multirow{3}{*}{$\begin{array}{l}\text { Basmacioglu et al (2004), Flourou-Paneri et } \\
\text { al (2005), Cross et al (2007), Fotea et al } \\
\text { (2009), Mathlouthi et al (2009) }\end{array}$} \\
\hline $\begin{array}{l}\text { Consommation } \\
\text { alimentaire }(\mathrm{g} / \mathrm{j})\end{array}$ & $79,2 \pm 9,4$ & $80,0 \pm 10,1$ & 0,665 & $+0,94$ & \\
\hline $\begin{array}{l}\text { Gain moyen } \\
\text { quotidien }(\mathrm{g} / \mathrm{j})\end{array}$ & $40,3 \pm 3,9$ & $42,0 \pm 4,5$ & 0,218 & $+4,11$ & \\
\hline
\end{tabular}

Les moyennes sont suivies par leurs déviations standards. 
protéines, lipides, cendres) dans les filets et la cuisse avec des doses de 0,1 , 0,3 ou $0,7 \%$ d'HE de thym. D'autres auteurs (Bolukbasi et al 2006) avaient observé, cependant, que la composition lipidique de la viande pouvait être modifiée à la dose de 100 ou 200 ppm. Ils décrivent une baisse des teneurs en Acides Gras (AG) saturés et polyinsaturés des lipides de la cuisse et du filet, au profit des AG mono-insaturés. Enfin, Abou-Sekken et al (2007) ont observé une diminution du gras abdominal avec l'utilisation de feuilles de thym chez le canard. Les différences de doses en « équivalent HE » d'une part, la nature même du produit d'autre part (feuilles entières, broyées, distillées...), et l'absence de détails sur la composition en principes actifs peuvent contribuer à expliquer ces contradictions.

La perception de la qualité sensorielle de la viande peut aussi être modifiée par l'emploi d'HE. Ainsi, avec l'HE de thym, l'odeur et la couleur de la viande des filets et des cuisses de poulet sont modifiées avec des doses de 0,1 à $0,7 \%$ dans l'aliment, la tendreté de la viande étant améliorée au seuil de $0,3 \%$, et la flaveur à partir de $0,7 \%$ (Fotea et al 2009).

Chez les pondeuses, Bolukbasi et al (2008) ont observé une amélioration de l'IC, expliquée par une diminution de la consommation alimentaire (-5\%) accompagnée d'une augmentation du taux de ponte $(+2,5 \%)$ et de la taille des œufs $(+10 \%)$. Le poids du jaune baisse, celui de la coquille augmente. Une diminution significative de la fraicheur a été enregistrée (- 3,4 unités Haugh), ce qui semble en contradiction avec l'effet protecteur de la coquille sur l'oxydation.

Denli et al (2004) ont identifié un intérêt de l'HE de thym pour les cailles de chair japonaises, en terme de baisse de 1 'IC, consécutive à une diminution de l'ingéré alimentaire.

D'une façon très générale, il semble que l'HE de thym améliore souvent l'IC chez plusieurs espèces avicoles. En revanche, les effets sur les autres paramètres zootechniques (GMQ, consommation d'aliment, composition corporelle...) sont plus aléatoires. Cette variabilité de réponses peut s'expliquer par la variabilité de la composition chimique (chémotypes) en principes actifs (tableau 1), rarement détaillée dans les publications.

\section{2 / L'huile essentielle d'origan}

Les effets de l'HE d'origan sur le poulet de chair ont fait l'objet de nombreux travaux publiés. Halle et al (2004) observent une amélioration significative de l'IC de 0,04 points liée à une baisse de la consommation alimentaire sans incidence sur la croissance. Tekeli et al (2006) et Basmacioglu et al (2010) observent que l'effet sur l'IC peut s'inverser à très forte dose.

D'autres auteurs (Basmacioglu et al 2004, Cross et al 2007, Symeon et al 2009) s'accordent sur l'absence d'effets significatifs sur la consommation, le GMQ ou l'IC, mais ces dernières études portaient aussi sur la physiologie des animaux, et ne disposaient donc pas d'un grand nombre de répétitions pour la mesure des performances de croissance.

La méta-analyse des effets de l'HE d'origan a permis de gagner de la puissance dans les analyses statistiques des effets de cette HE (tableau 4) et de conclure à une amélioration significative de l'IC de $1,8 \%$, principalement due à une amélioration significative de la croissance de $+2,4 \%$.

L'HE d'origan retarde l'oxydation des lipides dans la viande (Basmacioglu et al 2004). Des extraits d'origan peuvent stabiliser le tissu adipeux, les extraits de thym étant moins efficaces (Lee et al 2004). Par ailleurs, avec de l'HE d'origan, distribuée à raison de $250 \mathrm{mg} / \mathrm{kg}$ d'aliment, la qualité de la viande du poulet peut être modifiée en termes de couleur, de résistance au cisaillement et de pertes en eau à la cuisson à 9, 12 et 15 semaines (Symeon et al 2009). Un jury de dégustation n'a toutefois pas pu faire de distinction entre les différents échantillons (couleur, texture, flaveur).

Les effets de l'HE d'origan sur les performances des pondeuses sont moins renseignés. Tous les auteurs observent une amélioration du taux de ponte, une baisse de la consommation alimentaire et une amélioration de l'IC (Bampidis et al 2005, Florou-Paneri et al 2005, Radwan et al 2008). Ces différences ne sont pas toujours significatives, mais elles s'expriment toujours dans le même sens, ce qui conforte l'hypothèse d'un manque de puissance de ces essais. Mais le nombre de publications disponibles ne permet pas de réaliser une méta-analyse.

\section{3 / L'huile essentielle de romarin}

Comme pour le thym et l'origan, les effets de l'HE de romarin sur les performances du poulet de chair peuvent varier dans des sens opposés selon les auteurs : baisse du GMQ et augmentation de l'IC (Cross et al 2007) ; amélioration du GMQ et baisse de l'IC (Ghazalah et Ali 2008, Mathlouthi et al 2009). Enfin, d'autres études (Basmacioglu et al 2004, Flourou-Paneri et al 2005, Fotea et al 2009) ne rapportent pas d'effets significatifs.
La compilation de ces résultats via la méta-analyse met seulement en évidence une tendance $(P=0,066)$ à l'amélioration de l'IC (tableau 4).

La variabilité des teneurs en phénols de type carvacrol (ou cymophénol) et thymol (tableau 3) pourrait expliquer l'existence d'effets variables de l'HE de romarin d'un essai à l'autre. En effet, Lee et al (2003) observent des effets opposés selon qu'ils utilisent du carvacrol seul, lequel diminue la consommation alimentaire et le gain de poids mais améliore l'IC, ou du thymol seul, qui exerce un effet contraire et de moindre importance. Comme les études sur les HE de romarin ne précisent pas toujours les teneurs en carvacrol et en thymol, on peut émettre l'hypothèse que les effets supposés contradictoires d'une publication à l'autre s'expliquent par de variations importantes des teneurs en carvacrol et thymol des HE de romarin utilisées.

\section{4 / La toxicité des huiles essentielles}

\section{1 / Rappels de quelques grands principes de toxicologie}

a) Le principe de Paracelse : "c'est la dose qui fait le poison "

Parmi les grands précurseurs de la toxicologie moderne, le médecin suisse Paracelse (1494-1541) est le premier à avoir formellement relié la dangerosité d'un produit à la dose administrée : «Toutes les choses sont poison, et rien n'est sans poison; seule la dose fait qu'une chose n'est pas un poison ». Bien que certaines molécules tendent à échapper aujourd'hui à ce principe (cas des perturbateurs endocriniens), ce principe prévaut encore largement au sein de la communauté scientifique, servant de base à l'évaluation toxicologique de tous les produits susceptibles de générer des effets toxiques chez l'Homme ou l'animal.

\section{b) Notion de toxicité d'un produit}

La toxicité d'un principe actif est une notion très complexe. Décrire la « toxicité » d'un produit de façon précise sous-entend aborder les effets néfastes qu'il provoque dans différents cas de figures pour une espèce animale donnée, un mode d'administration donné (par voies orale, respiratoire, cutanée, oculaire, intra-veineuse...) et une dose donnée (principe de Paracelse). La dose administrée peut être soit "forte et unique » et on parle alors de « toxicité aigüe », soit « plus faible et répétée dans le temps » et on parle alors de « toxicité chronique » ou « subaigüe ». Afin d'être comparable d'une espèce animale à une 
Tableau 5. Paramètres toxicologiques du camphre définis sur animaux modèles et l'Homme (Source : US EPA, 2010).

\begin{tabular}{|l|l|}
\hline Toxicologie aigüe & DL 50*, voie orale, souris $1310 \mathrm{mg} / \mathrm{kg} \mathrm{PV}$ \\
\hline Toxicologie chronique & NOAEL ${ }^{* *}, 13$ semaines, voie orale, souris $250 \mathrm{mg} / \mathrm{kg} / \mathrm{j}$ \\
\hline Tératogénicité & NOAEL : $>1000 \mathrm{mg} / \mathrm{kg} / \mathrm{j}$ \\
\hline Toxicités fotale et maternelle & NOAEL : Lapin : $400 \mathrm{mg} / \mathrm{kg} / \mathrm{j}$ rat $>800 \mathrm{mg} / \mathrm{kg} / \mathrm{j}$ \\
\hline Toxicité aigüe & Dose létale suite à exposition (Homme) $: 15$ à $500 \mathrm{mg} / \mathrm{kg}$ PV \\
\hline
\end{tabular}

* DL 50 : Dose Létale 50 ; **NOAEL : « No Observable Adverse Effect Level »

autre, cette dose est toujours rapportée à 1 kilogramme de poids vif.

Les différents effets toxiques observés dans chacun des cas de figures peuvent aller d'une « simple perte de poids ou d'appétit» à la «mort de l'indivi$\mathrm{du}$ », en passant par des tableaux cliniques extrêmement variables tant par leur nature que par leur gravité. Décrire la toxicité d'un produit nécessitera alors la mise en œuvre d'un ensemble de tests standardisés (lignes directrices et protocoles disponibles sur le site internet de l'OCDE : www.oecd.org) et la description des différents types de manifestations toxiques d'un produit (mortalité : DL50, dose pour laquelle est observée une mortalité de $50 \%$, irritation, aller-

Figure 1. Exemple de démarche d'évaluation toxicologique d'un produit.

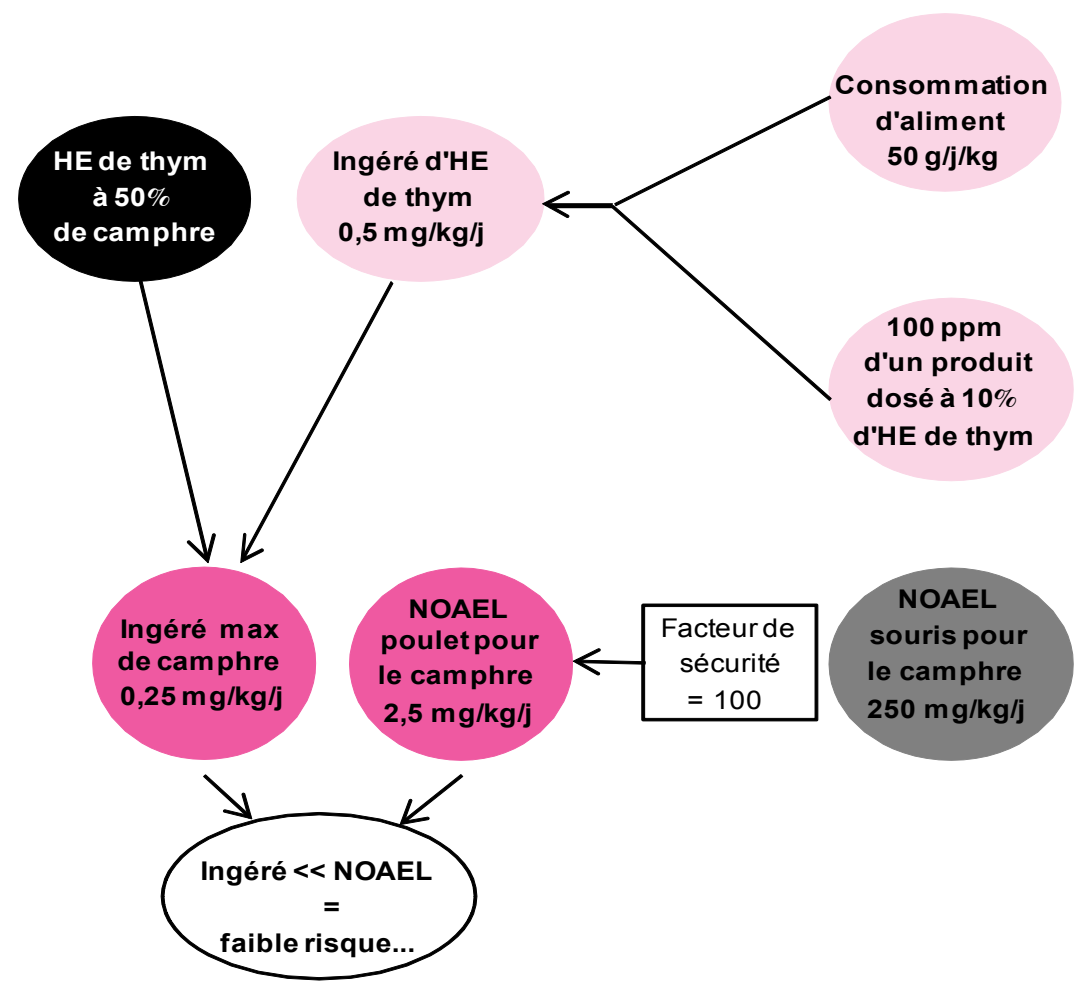

Hypothèses:

- Produit commercial dosé à 10\% d'une HE de thym contenant $50 \%$ de camphre.

- Le produit est ajouté dans l'aliment à 100 ppm.

- Evaluation de la toxicité basée sur le NOAEL « No Observable Adverse Effect

Level » souris.

- Facteur de sécurité de 100 (i.e. X 10 intra and X 10 entre espèces).
Le NOAEL (tableau 5) correspond à la dose la plus forte administrée à une espèce donnée, qui ne génère aucun effet toxique observable. On parle alors, par exemple, de «NOAEL Rat » ou de «NOAEL Souris ».

A partir de ce NOAEL, les autorités compétentes peuvent fixer une DJA. Elle est obtenue en appliquant plusieurs marges de sécurité successives au NOAEL, tenant compte des variabilités de réponses possibles, d'un animal à l'autre, et d'une espèce animale à une autre. En effet, la DJA (indicateur destiné à protéger l'Homme) est établie sur les bases de données toxicologiques recueillies chez l'animal. La DJA d'un principe actif est donc la dose à ne pas dépasser dans un aliment pour que le consommateur soit assuré de ne courir aucun risque, même en consommant cet aliment tous les jours de sa vie. Ajoutons que les DJA sont établies en tenant également compte des cas cliniques rapportés lors d'intoxications chez l'Homme.

\section{2 / Application aux huiles essen- tielles}

a) Le règlement (CE) $n^{\circ} 2003 / 1831$, garant de l'innocuité des produits

En Europe, le positionnement des HE en alimentation animale dans la catégorie des additifs (règlement (CE) $n^{\circ} 2003 /$ 1831) impose aux fabricants de constituer un dossier très complet (cf. $\S 1.3$ ). La première partie de ce dossier d'autorisation traite de la «Qualité » du produit, dans laquelle le fabricant a l'obligation de garantir la composition en ses principes actifs (cf. $\S 1.2$ ). Dans le cas des HE, on parle de produit «natureidentique ». La composition étant connue et constante (tout du moins dans des plages de variation connues et acceptées), la seconde partie du dossier d'autorisation (partie « Sécurité ») peut alors être présentée. Elle contient tous les éléments permettant de démontrer l'innocuité du produit pour l'Homme, l'animal et l'environnement. Ces travaux sont soit puisés dans la littérature scientifique, soit réalisés dans ce but en station expérimentale. Ainsi, même si le contenu de ces dossiers est rarement publié, on sait que l'innocuité de tout additif autorisé doit être démontrée aux autorités.

b) Les Huiles Essentielles incorporées dans les aliments présentent-elles des risques pour les volailles?

Compte tenu de la complexité des HE d'une part, et du concept de « toxicité » d'autre part, il est bien évidemment impossible de répondre formellement à cette question. Nous pouvons néanmoins, grâce à un exemple fictif simple, 
simuler une première approche de la toxicité d'un produit à base d'HE (figure 1). Cette démarche de simulation, basée sur des hypothèses extrêmes («worst case scenario ») est l'approche de référence permettant d'appréhender la toxicité d'un nouveau médicament vétérinaire. L'exemple choisi est un additif destiné au poulet de chair, comportant $10 \%$ d'une $\mathrm{HE}$ de thym particulièrement riche en camphre (50\%), composé dont les paramètres toxicologiques sont bien connus.

La démarche couramment appliquée pour l'évaluation toxicologique des molécules médicamenteuses consiste à appréhender la dose maximale à laquelle un individu peut être exposé, soit dans notre exemple, la dose ingérée quotidiennement par les animaux (figure 1 , sphères de couleur rose). En partant de l'hypothèse qu'un poulet de $2 \mathrm{~kg}$ de poids vif consomme chaque jour 100 grammes d'aliment supplémenté à 100 ppm avec notre produit contenant $10 \%$ d'HE de thym, on peut calculer que la dose d'HE de thym consommée est de $0,5 \mathrm{mg}$ par $\mathrm{kg}$ de poids vif et par jour.

Le camphre est une molécule potentiellement présente dans l'HE de thym. La teneur maximale observée dans la littérature est de $50 \%$ (sphère noire). Si on applique cette concentration au calcul précédent, la dose maximale consommée par notre volaille sera donc de $0,25 \mathrm{mg} / \mathrm{kg}$ de poids vif/jour. C'est cette dose qui doit maintenant être comparée aux données toxicologiques connues pour le camphre (sphère grise).

Pour extrapoler la valeur de « NOAEL souris » du camphre $(250 \mathrm{mg} / \mathrm{kg}$ de poids vif et par jour) à une autre espèce animale (ici la volaille), la règle couramment utilisée dans les évaluations toxicologiques de produits de médecine vétérinaire (EMEA 2005) est de considérer un facteur de sécurité de $\times 100$ (i.e. $\mathrm{X} 10$ pour palier le risque de variabilité interindividuelle et $X 10$ pour palier le risque de variabilité inter-spécifique). Dans notre exemple, et à la condition qu'aucun élément scientifique connu sur le camphre ne plaide en la faveur d'un facteur de sécurité plus élevé, un «NOAEL souris " de $250 \mathrm{mg} / \mathrm{kg}$ de poids vif et par jour mesuré chez la souris pour le camphre peut être extrapolé à la volaille à la valeur de $2,5 \mathrm{mg} / \mathrm{kg}$ de poids vif et par jour. Cette valeur de $2,5 \mathrm{mg} / \mathrm{kg}$ de poids vif est encore 10 fois supérieure à la dose potentiellement consommée par les volailles (sphères rouges). Dans notre exemple (un produit à base d' $\mathrm{HE}$ de thym riche en camphre), le risque d'observer un quelconque effet toxique chez les volailles est donc faible.

Bien entendu, cet exemple n'est pas suffisant en soi. En effet, les HE sont des produits complexes pour lesquels des interactions entre principes actifs ne sont pas à exclure. Cependant, il nous indique que les doses d'HE « additif » appliquées en alimentation des volailles sont probablement assez loin des doses pouvant entraîner des troubles chez les animaux. Enfin, ajoutons que des tests de tolérance sur espèce cible (au minimum jusqu'à 10 fois la dose recommandée) doivent être mis en œuvre pour compléter la démonstration de l'innocuité du produit.

\section{Conclusion}

Les HE, comme les extraits végétaux, font l'objet de nombreuses allégations, mais parmi les nombreux travaux publiés, les effets observés, aussi bien au niveau zootechnique, qu'à l'échelle d'un organe ou d'un tissu, sont très variables. La difficulté à mettre en évidence ces effets a essentiellement deux causes distinctes : le manque de puissance statistique des essais zootechniques disponibles dans la littérature, et la variabilité de la composition en principes actifs des produits testés.

En effet, les différences observées dans les essais zootechniques restent souvent faibles et nécessitent très certainement d'augmenter le nombre de répétitions. En particulier, 1'IC est souvent analysé avec moins de 10 répétitions, ce qui est insuffisant pour ce type de paramètre. L'approche par méta-analyse peut permettre de pallier ce manque de répétitions, à condition toutefois de disposer de suffisamment d'articles exploitables.

De plus, la notion même d'HE reste peu précise en termes de composition chimique. Les teneurs en principes actifs sont très variables et ces mêmes principes peuvent exercer des effets contraires au sein de la même HE. C'est cette raison qui a conduit beaucoup de fabricants à utiliser des principes actifs purs plutôt qu'une HE plus complexe en principes actifs.

L'utilisation d'HE pour améliorer les performances des volailles semble une voie envisageable. Elle pose toutefois la question de l'efficacité économique de cette solution et de la compréhension des mécanismes qui permettent ces améliorations.

\section{Remerciements}

Nous tenons tout particulièrement à remercier Monique Berger pour ses précieux conseils statistiques, et Sarah Guardia pour une partie des recherches bibliographiques de la méta-analyse.

\section{Références}

Abou-Sekken M.S., Moustafa K.E.M.E., Elalfy T.S., 2007. Effect of fennel, thyme and probiotic as feed additives on the performance and the microbial content of the intestine of Muscovy ducks. Egyp. Poultry Sci. J., 27, 1009-1029.

AFSSA, 2007. Propositions pour une démarche d'évaluation de substances ou de produits « nouveaux » destinés à l'alimentation animale. Cas particulier des substances et produits à base de plantes. Rapport du groupe de travail AFSSA «Substances et produits nouveaux, ou à effet nouveau revendiqué, destinés à l'alimentation des animaux ", Février 2007, 65p.

Al Kassie G.A.M., 2009. Influence of two plant extracts derived from thyme and cinna- mon on broiler performance. Pakistan Vet. J., 29, 169-173.

Azizi A., Yan F., Honermeier B., 2008. Herbage yield, essential oil content and composition of three oregano (Origanum vulgare L.) populations as affected by soil moisture regimes and nitrogen supply. Industrial Crops and Products, Allemagne, 29, 554-561.

Bampidis V.A., Christodoulou V., FlourouPaneri P., Christaki E., Chatzzopoulou P.S., Tsiligianni T., Spais A.B., 2005. Effect of dietary dried oregano leaves on growth performance, carcase characteristics and serum cholesterol of female early maturing turkeys. Br. Poultry Sci., 46, 595-601.

Basmacioglu H., Tokusoglu O., Ergul M., 2004. The effect of oregano and rosemary essential oils or alpha-tocopherol acetate on performance and lipid oxidation of meat enriched with n-3 PUFA's in broilers. South Afric. J. Anim. Sci., 34, 197-210.

Basmacioglu H., Baysal S., Misirlioglu Z., Polat M., Yilmaz H., Turan N., 2010. Effects of oregano essential oil with or without feed enzymes on growth performance, digestive enzyme, nutrient digestibility, lipid metabolism and immune response of broilers fed on wheat-soybean meal diets. Br. Poultry Sci., 51, 67-80.

Bolukbasi S.C., Erhan M.K., Ozkan A. 2006. Effect of dietary thyme oil and vitamin E on growth, lipid oxidation, meat fatty acid composition and serum lipoproteins of broilers. S. Afr. J. Anim. Sci., 36, 189-196. 
Bolukbasi S.C., Erhan M.K., Ozkan A., 2008. The effect of feeding thyme, sage and rosemary oil on laying hen performance, cholesterol and some proteins ration of egg yolk and Escherichia Coli in feces. Arch. Geflugelk., 72, 231-237.

Botsoglou N.A., Fletouris D.J., FlorouPaneri P., Christaki E., Spais A.B., 2003. Inhibition of lipid oxidation in long-term frozen stored chicken meat by dietary oregano essential oil and $\alpha$-tocopheryl acetate supplementation. Food Res. Int., 36, 207-213.

Bousbia N., Abert Vian M., Ferhat M.A., Petitcolas E., Meklati B., Chemat F., 2009. Comparison of two isolation methods for essential oil from rosemary leaves: Hydrodistillation and microwave hydrodiffusion and gravity. Food Chem., 114, 355-362.

Brenes A., Roura E., 2010. Essential oils in poultry nutrition: Main effects and modes of action. Anim. Feed Sci. Technol., 158, 1-14.

Commission européenne, 2003. Règlement (CE) $n^{\circ} 1831 / 2003$ du parlement européen et du conseil du 22 septembre 2003 relatif aux additifs destinés à l'alimentation des animaux.

Commission européenne, 2008. Règlement (CE) $n^{\circ} 429 / 2008$ de la commission du 25 avril 2008 relatif aux modalités d'application du règlement $(\mathrm{CE}) \mathrm{n}^{\circ} 1831 / 2003 \mathrm{du}$ parlement européen et du conseil en ce qui concerne l'établissement et la présentation des demandes ainsi que l'évaluation et l'autorisation des additifs pour l'alimentation animale.

Cross D.E., McDevitt R.M., Hillman K. Acamovic T., 2007. The effect of herbs and their associated essential oil on performance, dietary digestibility and gut microflora in chicken from 7 to 28 days of age. Br. Poultry Sci., 48, 496-506.

Demir E., Sarica S., Ozcan M.A., Suicmez M., 2005. The use of natural feed additives as alternative to an antibiotic growth promoter in broiler diets. Arch. Geflugelk, 69, 110-116.

Denli M., Okan F., Uluocak A.N., 2004 Effect of dietary supplementation of herb essential oil on the growth performance, carcass and intestinal characteristics of quail. South Afr. J. Anim. Sci., 34, 174-179.

Dorman H.J.D., Deans S.G., 2000. Antimicrobial agents from plants: antibacterial activity of plant volatile oils. J. Appl. Microbiol., 88, 308-316.

Elhusseiny O., Ghazalah A.A., Mehrez A.Z., 1980. Response of broilers to dietary selfselection. Poult. Sci., 59, 1603-1604.

EMEA, 2005. Guideline on user safety for pharmaceutical veterinary medicinal products. Guideline reference $n^{\circ}$ EMEA/CVMP/543/03FINAL.

Filippo d'Antuono L., Galletti G.C., Bocchini P., 2000. Variability of essential oil content and composition of Origanum vulgare $L$. populations from a North Mediterranean Area (Liguria Region, Nothern Italy). An. Botany, 86, 471-478.

Flourou-Paneri P., Nicolaskis I., Giannenas I., Koidis A., Botsoglou E., Dotas V., Mitsopoulos I., 2005. Hen performance and egg quality as affected by dietary oragano essential oil and tocopheryl acetate supplementation. Int. J. Poult. Sci., 4, 449-454.

Fotea L., Costachescu E., Hoha G., 2009. The effect of essential oil of rosemary (rosmarinus officinalis) on the broilers growing per- formances. Lucrari Stiintifice - Universitatea de Stiinte Agricole si Medicina Veterinara, Seria Zootehnie., 52, 111-113.

Ghazalah A.A., Ali A.M., 2008. Rosemary leaves as dietary supplement for growth in broiler chickens. Int. J. Poult. Sci., 7, 234239.

Giannenas I., Florou-Paneri P., Papazahariadou M., Christaki E., Botsoglou N.A., Spais A.B., 2003. Effect of dietary supplementation with oregano essential oil on performance of broilers after experimental infection with Eimeria Tenella. Arch. Anim. Nutr., 57, 99106.

Halle I., Thomann R., Bauermann U. Henning M., Kohler P., 2005. Effect of graded supplementation of herb and essential oils in broiler feed on growth and carcass traits. Proc. $15^{\text {th }}$ Eur. Symp. Poult. Nutr., Balatonfured, Hungary, 25-29 sept 2005, 279-281.

Imelouane B., Amhamdi H., Wathelet J.P., Ankit M., Khedid K., El Bachiri A., 2009. Chemical composition and antimicrobia activity of essential oil of thyme (Thymus vulgaris) from Eastern Morocco. Int. J. Agric. Biol., 11, 205-208.

Jamshidi R., Afzali Z., 2009. Chemical composition of hydrodistillation essential oil of rosemary in different origins in Iran and comparison with other countries. AmericanEurasian J. Agric. Environ. Sci., 5, 78-81.

Jang I.S., Ko Y.H., Kang S.Y., Lee C.Y., 2007. Effect of a commercial essential oil on growth performance, digestive enzyme activity and intestinal microflora population in broiler chickens. Anim. Feed Sci. Technol., 134, 304315 .

Konjufca V.H., Pesti G.M., Bakalli R.I., 1997. Modulation of cholesterol level in broiler meat by dietary garlic and copper. Poult Sci., 76, 1264-1271.

Konstantopoulou I., Vassilopoulou L., Mavragani-Tsipidou P., Scouras Z.G., 1992. Insecticidal effects of essential oils. A study of the effects of essential oils extracted from eleven Greek aromatic plants on Drosophilia auraria. Experientia, 48, 616-619.

Lee K.W., Everts H., Kappert H.J., Frehner M., Losa R., Beynen A.C., 2003. Effect of dietary essential oil components on growth performance, digestive enzymes and lipid metabolism in female broiler chickens. $\mathrm{Br}$. Poult. Sci., 44, 450-457.

Lee K.W., Everts H., Beynen A.C., 2004 Essential oils in broiler nutrition. Int. J. Poult. Sci., 3, 738-752.

Loomis W.D., Croteau R., 1980. Biochemistry of terpenoids. The biochemistry of plants. A comprehensive treatise. Lipids: structure and function, 4, 363-418.

Martino L., De Feo V., Formisano C. Mignola E., Senatore F., 2009. Chemical composition and antimicrobial activity of the essential oils from three chemotypes of Origanum vulgare L. ssp. hirtum (Link) Ietswaart growing wild in Campania (Southern Italy). Molecules, 14, 2735-2746.

Mathlouthi N., Bouzaienne T., Oueslati I., Recoquillay F., Hamdi M., Bergaoui R., 2009. Effet de deux préparations d'huiles essentielles sur la croissance des bactéries in vitro et les performances du poulet de chair. INRA (Eds). $8^{\text {emes }}$ Journ. Rech. Avicole, INRA St Malo, 454 458
Najafi P., Torki M., 2010. Performance, blood metabolites and immune-competence of broiler chicks fed diets included essential oils of medicinal herbs. J. Anim. Vet. Adv., 9, 1164-1168.

Pandey R., Kalra A., Tandon S., Mehrotra N., Singh H.N., Kumar S., 2000. Essential oil compounds as potent source of nematicidal compounds. J. Phytopathol., 148, 501-502.

Pharmacopée Européenne, 2011. $7^{\text {ème }}$ édition du $1^{\mathrm{er}}$ Juillet 2011 "Direction Européenne de la qualité du médicament et soins de santé " European directorate for the quality of medeci$n e$ and healf care.

Porte A., Godoy R., 2007. Chemical composition of Thymus vulgarius L. (thyme) essential oil from the Rio de Janeiro State (Brazil). J. Serbian Chem. Soc., 73, 307-310.

Radwan Nadia L., Hassan R.A., Qota E.M., Fayek H.M, 2008. Effect of natural antioxidant on oxidative stability of eggs and productive and reproductive performance of laying hens. Int. J. Poult. Sci., 7, 134-150.

Santoyo S., Cavero S., Jaime L., Ibañez E. Señoráns F.J., Reglero G., 2005. Chemical composition and antimicrobial activity of Rosmarinus officinalis $L$. essential oil obtained via supercritical fluid extraction. Food Protect., 68,790-795.

Sax N.I., 1984. Dangerous properties of industrial materials Van Nostrand Reinhold (Ed). $6^{\text {th }}$ Ed. New-York, USA, 3124p.

Smith T.J., George D.R., Sparagano O., Seal C., Shiel R.S., Guy J.H., 2009. A pilot study into the chemical and sensorial effect of thyme and pennyroyal essential oil on hens eggs. Int J. Food Sci. Technol., 44, 1836-1842.

Sokovic M.D., Vukojevic J., Marin P.D., Brkic D.D., Vajs V., Van Griensven L.J.L.D., 2009. Chemical composition of essential oils of thymus and mentha species and their antifungal activities. Molecules, 14, 238-249.

Soto Mendivil E.A., Moreno Rodriguez J.F., Espinosa M.E., Garcia Fajardo J.A., Obledo Vazquez E.N., 2006. Chemical composition and fungicidal activity of the essential oil of thymus vulgaris against alternaria citri. e-Gnosis [on line], 4, 1-7.

Symeon G.K., Zintilas C., Ayoutanti A., Bizelis J.A., Deligeorgis S.G., 2009. Effect of dietary oregano essential oil supplementation for an extensive fattening period on growth performance and breast meat quality of female medium-growing broilers. Can. J. Anim. Sci., 89, 331-334.

Tekeli A., Celik L., Kutlu H. R., Görgülü M., 2006. Effect of dietary supplemental plant extracts on performance, carcass characteristics, digestive system development, intestinal microflora and some blood parameters of broiler chicks. EPC 2006 - $12^{\text {th }}$ Eur. Poult. Conf., Verona, Italy, 10-14 September, 4-8.

Toghyani M., Tohidi M., Ali Gheisari A., Al Tabeidian S., 2010. Performance, immunity, serum biochemical and hematological parameters in broiler chicks fed dietary thyme as alternative for an antibiotic growth promoter. Afr. J. Biotechnol., 9, 6819-6825. 


\title{
Résumé
}

L'interdiction des antibiotiques comme facteur de croissance en élevage a dynamisé la recherche d'alternatives comme les extraits végétaux et en particulier les huiles essentielles. En aviculture, les huiles essentielles de thym, origan et romarin sont les plus étudiées. Leur composition en principes actifs est très variable, entraînant certainement une grande variabilité des réponses zootechniques. L'huile essentielle de thym semble favoriser le gain de poids, en particulier chez le poulet. Celle d'origan améliore l'indice de consommation et la vitesse de croissance chez le poulet de chair, et le taux de ponte et l'indice de consommation chez la poule pondeuse. Celle du romarin améliore aussi l'indice de consommation chez le poulet. Cependant, utilisées à forte dose, les huiles essentielles peuvent exercer des effets contraires. Toutefois, la consommation d'huiles essentielles aux doses utilisées (moins de $0,5 \mathrm{mg}$ par kg de poids vif) ne dépasse pas le seuil de toxicité pour l'animal ou pour le consommateur.

\begin{abstract}
Essential oils in poultry feeding. 1. Growth performances and regulation aspects

Due to the ban of antibiotic growth promoters, the use of essential oils in animal husbandry has been very much developed. In poultry feeding, essential oils produced from thyme, oregano and rosemary are the most studied. A noteworthy characteristic of essential oils is that the balance between the different active principles can be altered from one oil to another. Depending on the research that has been done, the effects of these essential oils can change a lot. Concerning thyme essential oil, guaranteeing a significant effect on growth performances is difficult; however, there is a tendency for a higher daily weight gain. As for oregano essential oil, food conversion ratio and growth performances are improved, as well as laying rates and feed intake for hens. Rosemary essential oil also seems to improve food conversion ratio. However, when used at excessive doses, essential oils can have adverse effects (an increase of feed conversion ratio in broilers and fall of global weight gain). But, on the basis that a broiler eats $0.5 \mathrm{mg}$ of essential oil per kilogram of live weight per day, the toxic risk is very low, both for the broiler and for humans.
\end{abstract}

ALLEMAN F., GABRIEL I., DUFOURCQ V., PERRIN F., GABARROU J.-F., 2013. Utilisation des huiles essentielles en alimentation des volailles. 1. Performances de croissance et règlementation. INRA Prod. Anim., 26, 1, 3-12. 